J. Beerten, S. Cole, and R. Belmans, "Modeling of multi-terminal VSC HVDC systems with distributed DC voltage control," IEEE Transactions on Power Systems, vol. 29, no. 1, pp. 34-42, Jan. 2014.

Digital Object Identifier: 10.1109/TPWRS.2013.2279268

URL:

http://ieeexplore.ieee.org/xpl/articleDetails.jsp?tp=\&arnumber=6588621

( 2013 IEEE. Personal use of this material is permitted. Permission from IEEE must be obtained for all other users, including reprinting/ republishing this material for advertising or promotional purposes, creating new collective works for resale or redistribution to servers or lists, or reuse of any copyrighted components of this work in other works. 


\title{
Modelling of Multi-terminal VSC HVDC Systems with Distributed DC Voltage Control
}

\author{
Jef Beerten, Member, IEEE, Stijn Cole, Member, IEEE, and Ronnie Belmans, Fellow, IEEE
}

\begin{abstract}
This paper discusses the extension of electromechanical stability models of Voltage Source Converter High Voltage Direct Current (VSC HVDC) to Multi-terminal (MTDC) systems. The paper introduces a control model with a cascaded DC voltage control at every converter that allows a two-terminal VSC HVDC system to cope with converter outages. When extended to a MTDC system, the model naturally evolves into a master-slave set-up with converters taking over the DC voltage control in case the DC voltage controlling converter fails. It is shown that the model can be used to include a voltage droop control to share the power imbalance after a contingency in the DC system amongst the converters in the system. Finally, the paper discusses two possible model reductions, in line with the assumptions made in transient stability modelling. The control algorithms and VSC HVDC systems have been implemented using both MatDyn, an open source MATLAB transient stability program, as well as the commercial power system simulation package EUROSTAG.
\end{abstract}

Index Terms-HVDC transmission control, Power system modeling, Power system stability.

\section{INTRODUCTION}

$\mathbf{O}$ VER the last decade, the power engineering world is showing an increasing interest in Voltage Source Converter High Voltage Direct Current (VSC HVDC) technology. In Europe, suggestions have even been made to construct a new overlay DC grid based on VSC HVDC technology [1]. With these prospects of extending the principles of VSC HVDC to Multi-terminal (MTDC) configurations, the modelling and control of MTDC systems has become one of the more prominent research topics. This paper introduces a generic electromechanical stability model for MTDC systems with a distributed DC voltage control. The focus of the paper is on the DC system itself and not on the interconnected AC/DC system.

When modelling the MTDC system, a distinction is traditionally made based on the level of modelling detail. ElectroMagnetic Transient Programs (EMTP) accurately represent the switching dynamics and electromagnetic transients. Averaged models and electromechanical stability models [2] have been used to study alternative outer controller structures [3]-[5], and optimized control settings [6]-[8] as well as dynamic

Jef Beerten is funded by a research grant from the Research Foundation Flanders (FWO).

Jef Beerten and Ronnie Belmans are with the Department of Electrical Engineering (ESAT), Division ELECTA, University of Leuven (KU Leuven), Kasteelpark Arenberg 10, bus 2445, 3001 Leuven-Heverlee, Belgium. (e-mail: jef.beerten@esat.kuleuven.be, ronnie.belmans@esat.kuleuven.be)

Stijn Cole is with the Power System Consulting group of Tractebel Engineering, Arianelaan 7, 1200 Brussels, Belgium (e-mail: stijn.cole@gdfsuez.com). interaction with the AC system [9], [10] and system frequency support [11], [12]. Power flow algorithms, as presented in [13], have been used to address the steady-state effects of a distributed DC voltage control [14], [15].

Significant work has already been carried out on the modelling and control of MTDC systems. With the DC system voltage being the most crucial control variable, most focus has been dedicated towards a distributed control of the DC voltage at different converters. The two main control methods are Voltage Margin Control [5], [16] and DC voltage droop control [6]-[8], [10], [17].

This paper builds upon the fundamental frequency modeling approach presented in [2]. Two important extensions are added to the model. Firstly, current and voltage limits are represented in detail in the current control loop and in the outer controller. Secondly, a cascaded control structure is introduced in the outer controller which allows power controlling converters to take over the voltage control when the DC voltage controlling converter fails. The main innovation is that this cascaded control structure for a two-terminal system, developed in the framework of this paper, is extended in a systematic way to obtain a generalized cascaded control scheme for MTDC systems. This generalized cascaded control scheme can accommodate for voltage margin control as well as for voltage droop control. The second contribution of the paper is the investigation of the effect of the detailed modeling of the current and voltage limits, by comparing the detailed model with a simplified model. The model has been developed and and implemented in MatDyn [18] at KU Leuven and implemented and tested in EUROSTAG [19] at Tractebel Engineering.

Sections II and III respectively provide a brief overview of the converter and DC grid model and the control structure for a two-terminal VSC HVDC system. In section IV, this structure is extended to a MTDC system, resulting in a voltage margin approach. Section IV also discusses the inclusion of a voltage droop in the control structure, enabling power sharing amongst different converters in case of a converter outage. Section V analyzes reduced order models and compares the results with those of the full model derived in the paper.

\section{CONVERTER AND DC GRID MODELLING}

The converter can be modelled as a controllable voltage source $\underline{u}_{c}$ behind a complex impedance $\underline{Z}_{c}=R_{c}+\jmath X_{c}$ connected to the Point of Common Coupling (PCC), as shown in Fig. 1. This complex impedance comprises both the converter reactance and the transformer. 


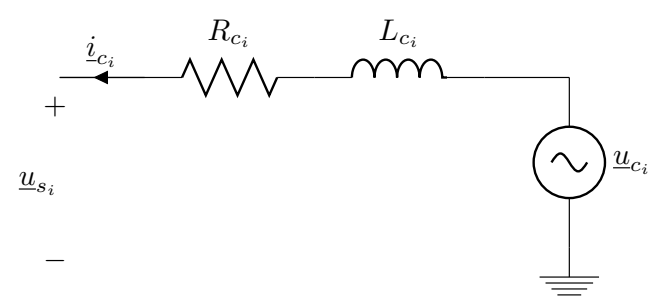

Fig. 1. Single phase diagram converter station AC side.

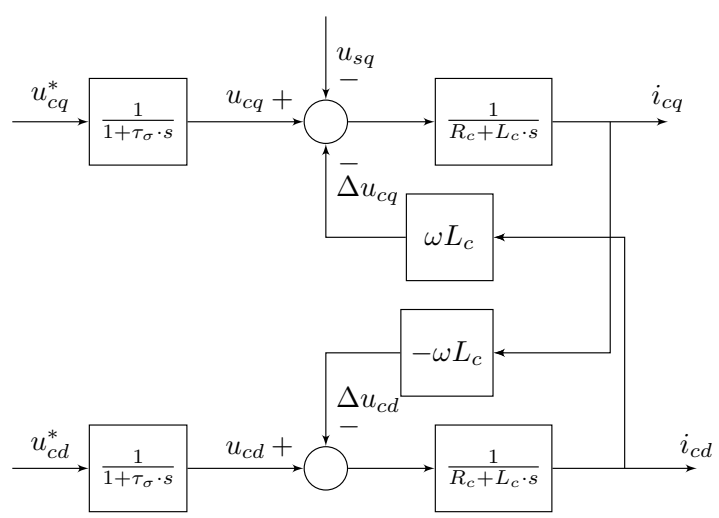

Fig. 2. Converter model block diagram.

Transforming the three-phase equations to a rotating $d q$ reference frame and assuming the grid voltage $\underline{u}_{s}$ to be entirely oriented in the $q$-direction, the converter equations become

$$
\begin{aligned}
& R_{c} i_{c q}+L_{c} \frac{d i_{c q}}{d t}=u_{c q}-\omega L_{c} i_{c d}-u_{s q}, \\
& R_{c} i_{c d}+L_{c} \frac{d i_{c d}}{d t}=u_{c d}+\omega L_{c} i_{c q} .
\end{aligned}
$$

The assumption that the voltage at the PCC is entirely aligned with the $q$-axis comes down to neglecting the effect of the phase-locked loop (PLL).

A first order system models the time delay caused by the processing and computation of the data and switching of the converter power electronics

$$
u_{c q}+\tau_{\sigma} \frac{d u_{c q}}{d t}=u_{c q}^{*} .
$$

A similar expression holds for $u_{c d}$. Fig. 2 schematically depicts the model.

The DC lines are represented by a lumped $\pi$-equivalent scheme, as depicted in Fig. 3. The DC voltage dynamics at bus $i$ are determined by [2]

$$
\begin{aligned}
C_{d c_{i}} \frac{d u_{d c_{i}}}{d t} & =i_{d c_{i}}+\sum_{j=1}^{i-1} i_{d c_{i j}}-\sum_{j=i+1}^{N} i_{d c_{i j}}, \\
\text { with } C_{d c_{i}} & =C_{d c, c_{i}}+\sum_{j=1}^{N} \frac{C_{d c_{i j}}}{2},
\end{aligned}
$$

with $u_{d c_{i}}$ and $i_{d c_{i}}$ respectively the DC voltage and current at bus $i, C_{d c, c_{i}}$ the converter DC capacitance, $i_{d c_{i j}}$ the current in the branch between buses $i$ and $j$ and $C_{d c_{i j}}$ the branch capacitance.

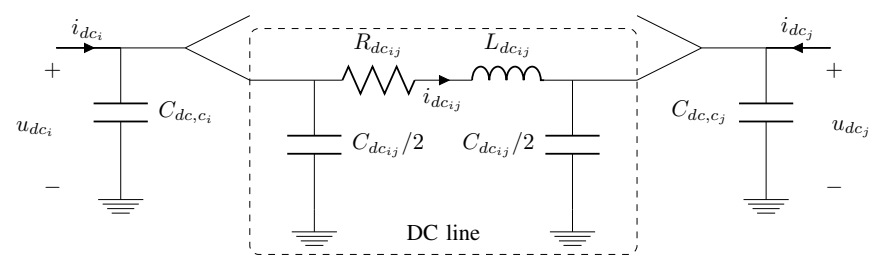

Fig. 3. DC side lumped parameter model.

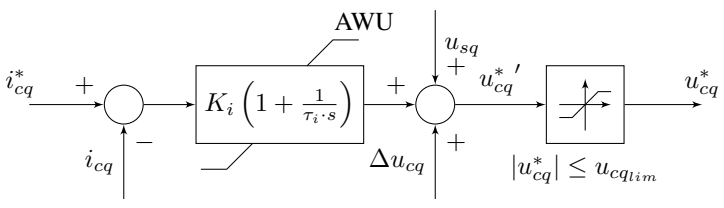

(a) $i_{c q}$ current controller

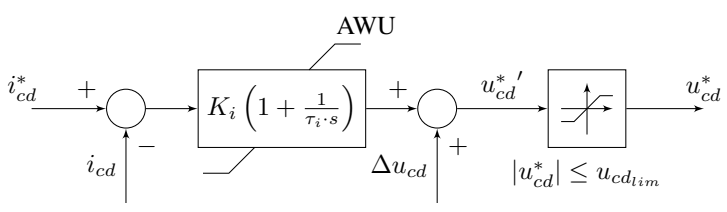

(b) $i_{c d}$ current controller

Fig. 4. Decoupled inner current controllers.

When the DC current dynamics are taken into account by modelling lumped inductances as shown in Fig. 3, the current dynamics of the branches connected to bus $i$ are modelled by

$$
L_{d c_{i j}} \frac{d i_{d c_{i j}}}{d t}+R_{d c_{i j}} i_{d c_{i j}}=u_{d c_{i}}-u_{d c_{j}}, \quad \forall 1 \leq j \leq N,
$$

with $R_{d c_{i j}}$ and $L_{d c_{i j}}$ the DC branch resistance and inductance. When the DC current dynamics are neglected, as discussed in section $\mathrm{V}$, the currents are eliminated as state variables.

\section{TWO-TERMINAL VSC HVDC CONTROL}

This section recapitulates the control of a two-terminal scheme, a full detailed description can be found in [20]. The first part briefly summarizes the decoupled current control principles, with emphasis on the converter voltage limits. The second part discusses different outer control loops. The third part proposes an alternative implementation using a cascaded structure of an active power controller and DC voltage controllers at the two converters in order to increase overall redundancy.

\section{A. Decoupled dq current control}

The VSC is controlled in a rotating $d q$-reference frame that is synchronized with the system voltage. Fig. 4 shows the inner current controllers, including an anti-windup (AWU).

The voltage limits $u_{c q_{l i m}}$ and $u_{c d_{l i m}}$ are determined by the maximum modulation factor $m_{\max }$ and the DC voltage $u_{d c}$. The maximum converter voltage magnitude $u_{c_{l i m}}$ can thus be written as

$$
u_{c_{l i m}}=m_{\max } u_{d c} .
$$


The limits can be implemented such that the controller can give priority to active or reactive power control. With the decoupling terms defined as

$$
\begin{aligned}
& \Delta u_{c q}=\omega L_{c} i_{c d}, \\
& \Delta u_{c d}=-\omega L_{c} i_{c q},
\end{aligned}
$$

a modified $q$-decoupling term can be defined as

$$
\Delta u_{c q}^{+}=\Delta u_{c q}+u_{s q} .
$$

When under voltage limitation, the terms $\Delta u_{c d}$ and $\Delta u_{c q}^{+}$ are prioritized over the voltages that are used to control the currents. When prioritizing active over reactive power control, $u_{c q_{l i m}}$, the $q$-limit can be written as

$$
u_{c q_{l i m}}= \begin{cases}\sqrt{u_{c_{l i m}}^{2}-\Delta u_{c d}^{2}} & \text { if }\left|\Delta \underline{u}_{c}^{+}\right| \leq u_{c_{l i m}} \\ \frac{u_{c_{l i m}}}{\sqrt{1+\left(\frac{\Delta u_{c d}}{\Delta u_{c q}^{+}}\right)^{2}}} & \text { if }\left|\Delta \underline{u}_{c}^{+}\right|>u_{c l i m}\end{cases}
$$

with the modified decoupling vector defined as $\Delta \underline{u}_{c}^{+}=$ $\Delta u_{c d}+\jmath \Delta u_{c q}^{+}$. The voltage limit in the $d$-axis, $u_{c d_{l i m}}$, can consequentially be expressed as

$$
u_{c d_{l i m}}=\sqrt{u_{c_{l i m}}^{2}-u_{c q}^{*}} \text {. }
$$

Alternatively, equal priority can be given to both $d$-and $q$ components by having

$$
\begin{aligned}
& u_{c q_{l i m}}=\frac{u_{c_{l i m}}}{\sqrt{1+\left(\frac{u_{c d}^{*^{\prime}}}{u_{c q}^{* \prime}}\right)^{2}}} \text { if }\left|\underline{u}_{c}^{* \prime}\right|>u_{c_{l i m}}, \\
& u_{c d_{l i m}}=\frac{u_{c_{l i m}}}{\sqrt{1+\left(\frac{u_{c q}^{* \prime}}{u_{c d}^{* \prime}}\right)^{2}}} \text { if }\left|\underline{u}_{c}^{* \prime}\right|>u_{c_{l i m}},
\end{aligned}
$$

when under voltage limitations. In these expressions, $\underline{u}_{c}^{* \prime}=$ $u_{c d}^{*}{ }^{\prime}+\jmath u_{c q}^{* \prime}$ is the value of the converter voltage references before limiting, as shown in Fig. 4.

\section{B. Standard two-terminal outer control}

The current control components $i_{c q}$ and $i_{c d}$ are physically linked to the active and reactive power that the VSC injects in the AC system. Fig. 5 shows the outer active and reactive power PI-controllers. As an alternative to the reactive power controller shown in Fig. 5b, one can use the $d$-axis current to directly control the voltage at the AC terminal. Reactive power control is not the main focus of this paper and will not be discussed further.

In a two-terminal scheme, one converters controls the active power (Fig. 5a) whereas the other controls the DC voltage at its DC bus (Fig. 6). When giving priority to active power over reactive power control, the current $q$ - and $d$-limits, respectively $i_{c q_{l i m}}$ and $i_{c d_{l i m}}$ in Figs. 5-6, are given by

$$
\begin{aligned}
& i_{c q_{l i m}}=i_{c_{l i m}}, \\
& i_{c d_{l i m}}=\sqrt{i_{c_{l i m}}^{2}-i_{c q}^{*}{ }^{2}} .
\end{aligned}
$$

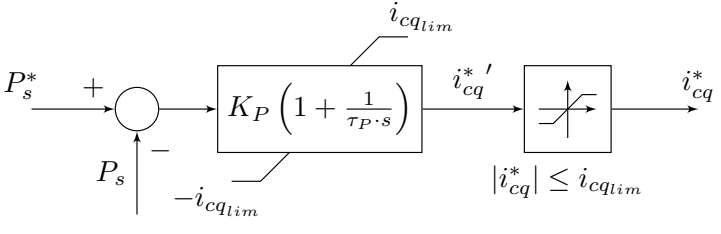

(a) Constant $P_{s}$ controller

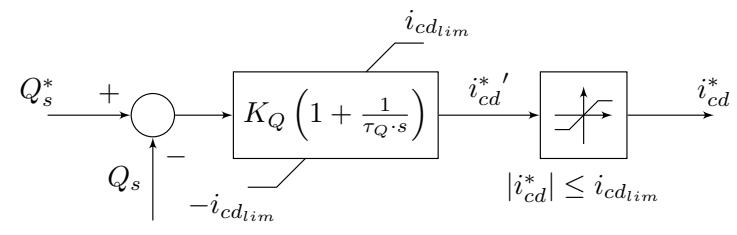

(b) Constant $Q_{s}$ controller

Fig. 5. Outer active and reactive power controllers.

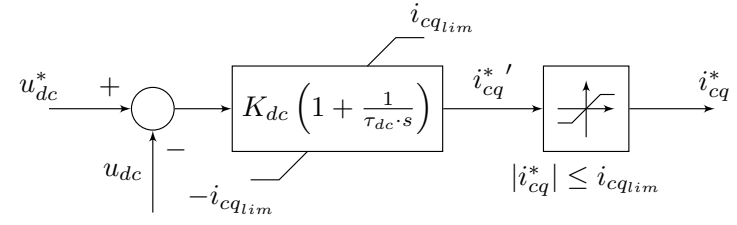

Fig. 6. Outer DC voltage controller.

Alternatively, equal priority can be given to the active and reactive power control, by having

$$
\begin{aligned}
& i_{c q_{l i m}}=\frac{i_{c_{l i m}}}{\sqrt{1+\left(\frac{i_{c d}^{*^{\prime}}}{i_{i q}^{* \prime}}\right)^{2}}} \text { if }\left|\underline{i}_{c}^{* \prime}\right|>i_{c_{l i m}}, \\
& i_{c d_{l i m}}=\frac{i_{c_{l i m}}}{\sqrt{1+\left(\frac{i_{c q}^{*^{\prime}}}{i_{c d}^{*}}\right)^{2}}} \text { if }\left|\underline{i}_{c}^{* \prime}\right|>i_{c_{l i m}},
\end{aligned}
$$

with $\underline{i}_{c}^{* \prime}=i_{c d}^{*}+j i_{c q}^{*}{ }^{\prime}$ the current reference before limiting, as shown in Figs. $5-6$. Instead of giving equal priority to both control components, this formulation can be generalized to prioritize one current component over the other, without completely compromising the other, by defining a constant ratio $\alpha$ such that

$$
\begin{aligned}
& i_{c q_{l i m}}=\frac{i_{c_{l i m}}}{\sqrt{1+\alpha^{2}}} \text { if }\left|\underline{i}_{c}^{* \prime}\right|>i_{c_{l i m}}, \\
& i_{c d_{l i m}}=\frac{i_{c_{l i m}}}{\sqrt{1+\left(\frac{1}{\alpha}\right)^{2}}} \text { if }\left|\underline{i}_{c}^{* \prime}\right|>i_{c_{l i m}} .
\end{aligned}
$$

When observing the steady-state behavior of a converter when under current limits, (15) - (16) results in the absence of any reactive power injected by the converter. The approach from (19) - (20) results in an operation at constant power factor $\cos \phi_{c}$ such that

$$
\cos \phi_{c}=\frac{1}{\sqrt{1+\alpha^{2}}} .
$$

This implementation guarantees operation at constant power factor and can be of interest when the reactive power support provided to the AC network has to be guaranteed when a 


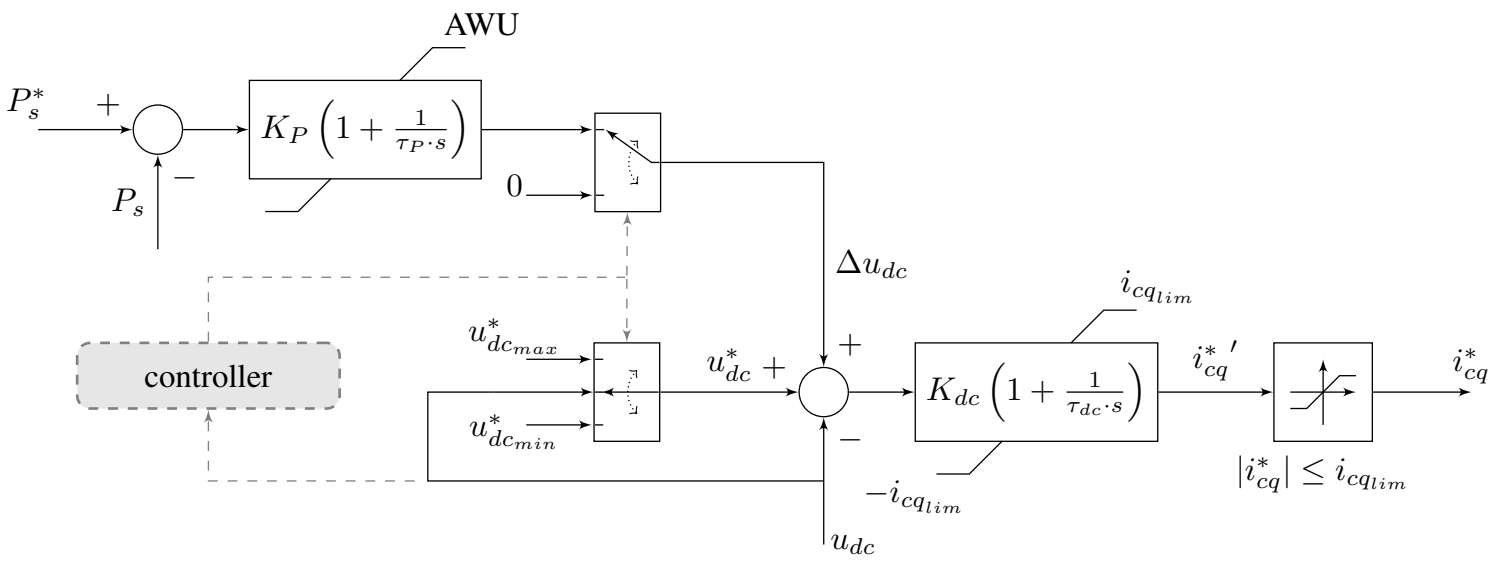

Fig. 7. Combined active power and DC voltage controller.

converter limit is hit. The implementation from (15) - (16) prioritizes active power, which makes it a suitable candidate for use in a DC voltage controlling converter. The steady-state behavior resulting from (17) - (18) on the contrary, depends on the values of the current reference before limiting $\underline{i}_{c}^{* \prime}$.

The reference currents are also reduced under $\mathrm{AC}$ fault conditions, to limit the short circuit contribution by the converter [21]. This can be easily included by having $i_{c, d q_{l i m}}=$ $i_{c, d q_{l i m, S C}}$ when the voltage at the PCC drops. Instead of limiting the currents under fault conditions, the priority can also be shifted to reactive power control to fulfill the grid code requirements concerning voltage support [22].

\section{Redundant outer control}

One of the disadvantages of the control implementation from the previous part, is that the control structure as such cannot cope with an outage or blocking of the DC voltage controlling converter. Whereas an outage or blocking of the power controlling converter only causes the power to drop, it does not cause a system outage since the DC voltage controlling converter can still control the DC voltage.

As the control of the DC voltage is crucial to the operation of the power system, one can therefore duplicate the DC voltage control, as proposed in [21] and elaborated in [23] for the power synchronization control, and mentioned in [24] for the operation of a two-terminal scheme. Fig. 7 shows the control structure for such a cascaded power control that has been developed in the framework of this paper. By implementing a correct control structure depending on the DC voltage at the converter's DC terminal, it is guaranteed that only one converter at a time controls the DC voltage.

In the power controlling converter, the DC voltage is used both as a reference signal and feedback signal as shown in Fig. 7, hence only $\Delta u_{d c}$ is retained as an input to the DC voltage controller. By using the actual DC voltage $u_{d c}$ instead of a reference value $u_{d c}^{*}$, one avoids counteracting actions of the DC voltage controller when the DC voltage varies as a result of an active power or DC voltage set-point change at another converter in the system.

The controller ensures that the power is controlled as long as the voltage stays within the limits $u_{d c_{\text {min }}}^{h i}$ and $u_{d c_{\text {max }}}^{l o}$. As soon

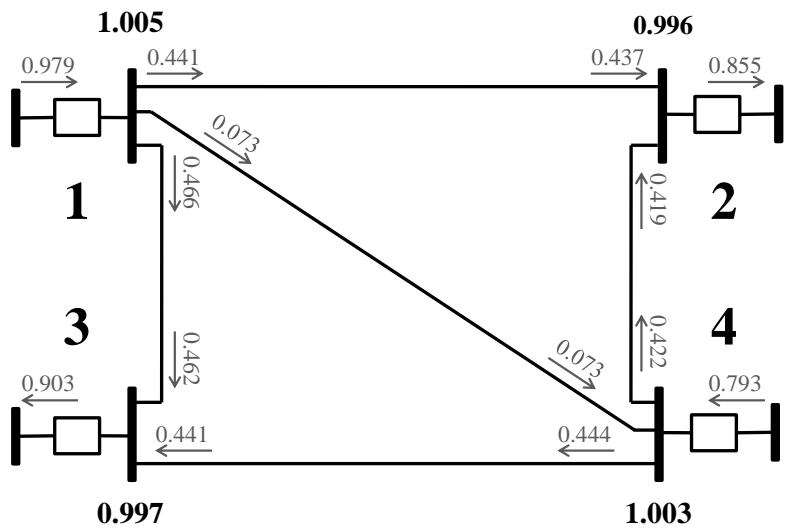

Fig. 8. Initial conditions of the test system.

as the voltage drops below $u_{d c_{\text {min }}}^{h i}$ or raises above $u_{d c_{\max }}^{l o}$, the controller switches to voltage control, controlling the voltage to one of the reference values, respectively $u_{d c_{\text {min }}}^{*}$ and $u_{d c_{\text {max }}}^{*}$. This is expressed mathematically as

$$
u_{d c}^{*}=\left\{\begin{array}{cll}
u_{d c_{\min }}^{*} & \text { if } & u_{d c} \leq u_{d c_{\min }}^{h i} \\
u_{d c} & \text { if } & u_{d c_{\min } i}^{h i}<u_{d c}<u_{d c_{\max }}^{l o} \\
u_{d c_{\max }}^{*} & \text { if } & u_{d c} \geq u_{d c_{\max }}^{l o}
\end{array}\right.
$$

with $u_{d c_{\min }}^{h i}=u_{d c_{\min }}^{*}+B_{d c}$ and $u_{d c_{\max }}^{l o}=u_{d c_{\max }}^{*}-B_{d c}$ and $B_{d c}$ a voltage deadband. As soon as the DC voltage reference changes to either $u_{d c_{\min }}^{*}$ or $u_{d c_{\max }}^{*}, \Delta u_{d c}$ is set to zero and the AWU of the active power PI-controller is activated to avoid an overshoot when the control switches back to active power mode. The deadband $B_{d c}$ prevents oscillating between the two different operational regimes. The DC voltage can start increasing or decreasing as a result of a power outage of the DC voltage controlling converter. By providing a cascaded structure with an inner DC voltage control as described above, a backup control is provided in case the DC voltage controlling converter fails. The remaining converter can now continue to operate as a STATCOM. In the next section, this cascaded general control structure will be used in a MTDC network. 


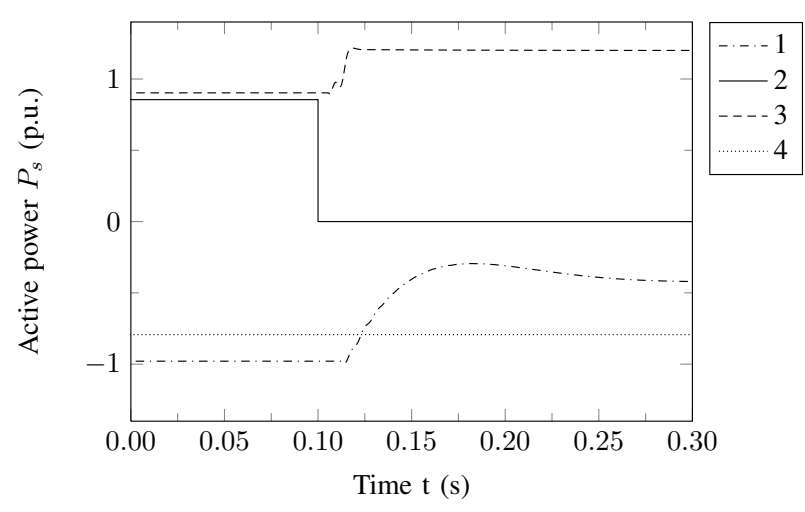

(a)

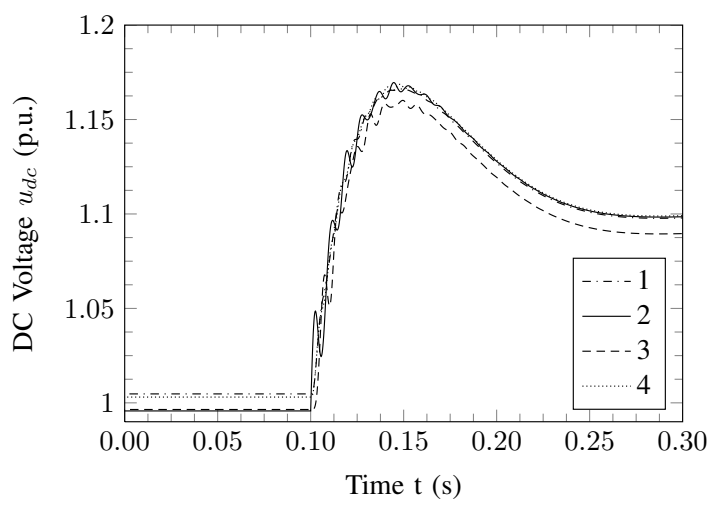

(b)

Fig. 9. Interactions of converters in the DC grid after outage of converter 2: (a) Active power $P_{s}$ to the AC grid and (b) DC voltage $u_{d c}$ (MatDyn simulation, including $L_{d c}$ ).

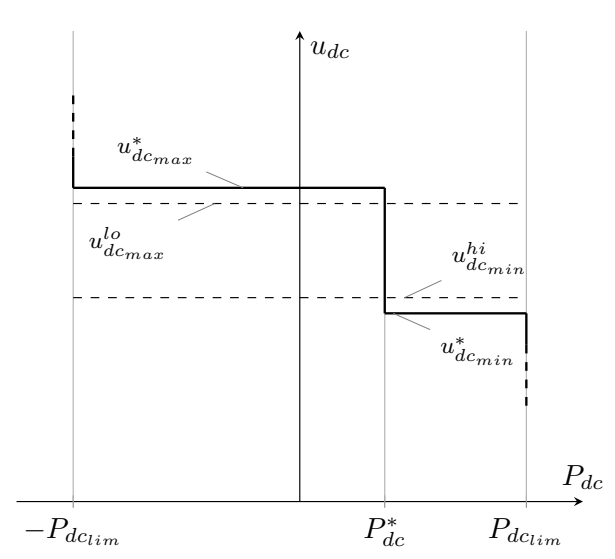

Fig. 10. Voltage margin control: P-V diagram.

\section{Multi-Terminal VSC HVDC CONTROL}

\section{A. Voltage margin control}

When the redundant control structure from the previous section is used in a multi-terminal configuration, it operates as a so called Voltage margin control [5] scheme, providing a backup DC slack converter in case the main slack converter (or DC voltage controlling converter) fails. Fig. 10 shows the steady-state $\mathrm{P}-\mathrm{V}$ characteristics resulting from the control implementation as observed from the DC side, thereby neglecting the converter losses. $P_{d c_{l i m}}$ is the active power limit resulting from the $i_{c q}$ limit discussed in section III. Consequently, the actual value of $P_{d c_{l i m}}$ depends on the voltage at the AC bus and the $i_{c q_{l i m}} / i_{c d_{l i m}}$ ratio. The voltage margin at different converters can be determined such that different converters can take over the DC voltage control, given that only one converter at a time can control the DC voltage.

Contrary to the two-terminal system, it is still possible to transfer power in case the DC slack converter fails or blocks. Fig. 9 shows simulation results for the voltage margin control implemented in the 4-terminal VSC HVDC system from Fig. 8 using MatDyn, an open-source MATLAB-based transient stability program [18]. A modified Euler ODE solver was used with a step size of 2e-4 s. The initial power flow solution from Fig. 8 , with the DC slack converter at bus 2 , has been obtained

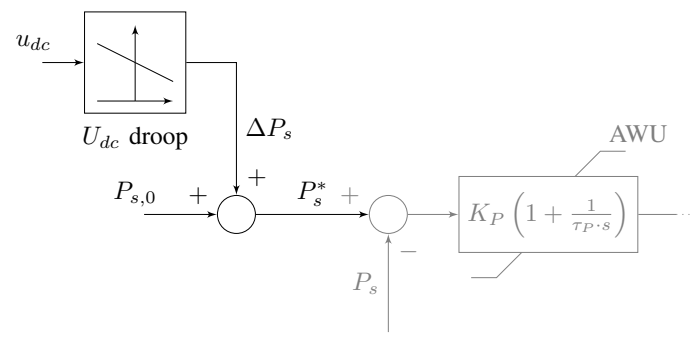

Fig. 11. DC voltage droop control.

using MATACDC, an open-source MATLAB-based AC/DC power flow program [25]. The power flow has been initialized such that the average voltage is equal to unity. After the outage of the DC voltage controlling converter 2, converter 3 initially tries to control the converter voltage when its converter upper voltage limit $u_{d c_{\max }}$ is reached. Since its current limit is hit, converter 3 is unable to control the converter voltage further, resulting in a further increase of the DC system voltage, after which converter 1 takes over the voltage control. The power in converter 4 remains unchanged because the influence of the changing DC voltage is not fed back to the power controlling converters as long as the voltage limits are not hit (Fig. 7).

\section{B. Voltage droop control}

Alternatively, the voltage control can be distributed amongst different converters by implementing a voltage droop control [17]. Fig. 11 shows how the droop control can be integrated in the redundant control structure from Fig. 7 (shown in gray). Contrary to standard droop control schemes presented in literature, which do not contain an inner DC voltage control loop, cascading the droop control as done in this paper has the advantage that upper and lower DC voltage limits can still be included as for 2-terminal schemes, ensuring that the DC voltage can still be controlled in case a converter is operating in islanding mode, e.g. as a result of DC breaker actions. The DC droop control can be implemented by letting

$$
P_{s}^{*}=P_{s, 0}+\frac{1}{k_{d c}}\left(u_{d c}-u_{d c, 0}\right)
$$




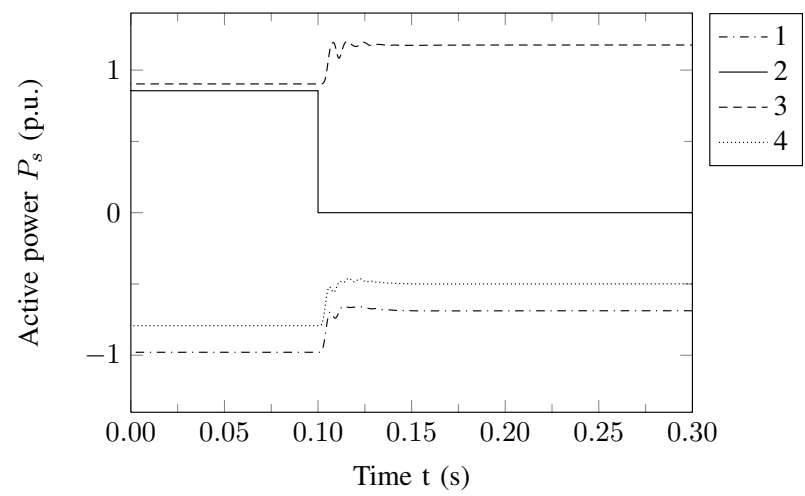

(a)

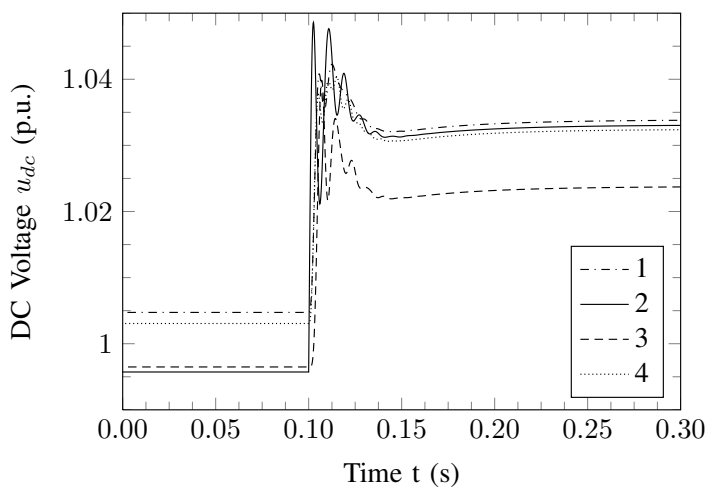

(b)

Fig. 12. Interactions of converters in the DC grid after outage of converter 2 (Voltage droop control): (a) Active power $P_{S}$ to the AC grid and (b) DC voltage $u_{d c}$ (MatDyn simulation, including $L_{d c}$ ).

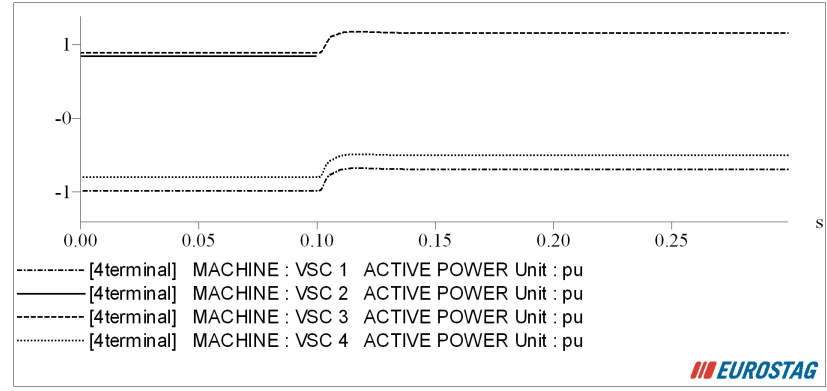

(a)

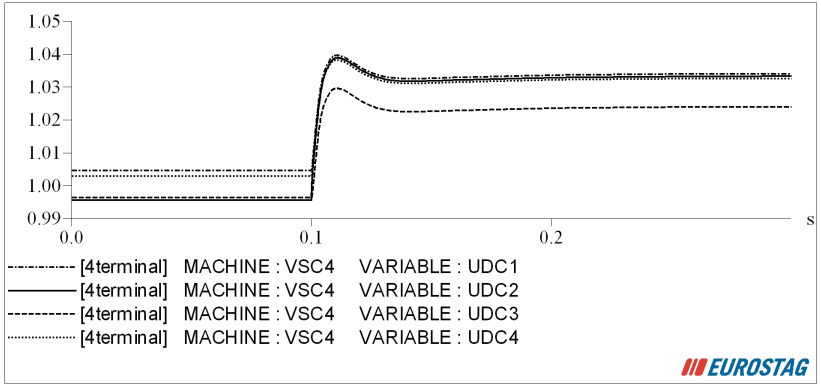

(b)

Fig. 13. Interactions of converters in the DC grid after outage of converter 2 (Voltage droop control): (a) Active power $P_{s}$ to the AC grid and (b) DC voltage $u_{d c}$ (EUROSTAG simulations, not including $L_{d c}$ ).

with $P_{s}^{*}$, the reference active power, depending on the set points of active power $P_{s, 0}$ and DC voltage $u_{d c, 0}$ and the droop factor $k_{d c}$. In [6], an alternative approach has been proposed to use an adaptive droop control based on a common voltage feedback signal instead of a local voltage measurement, thereby requiring communication. The droop values can be optimized taking into account the DC system dynamics, as done in [8] or both the AC and DC system dynamics, as in [6]. Fig. 14 shows the steady-state characteristics of the DC voltage droop when implemented as shown in Fig. 11. Similarly to the voltage margin control, $P_{d c_{l i m}}$ results from the $i_{c q}$-limit. The $u_{d c_{\text {min }}}$ and $u_{d c_{\max }}$ limits can be included at some converter stations to prevent the voltage from decreasing or increasing at a certain point. By setting $u_{d c_{\text {min }}}$ and $u_{d c_{\max }}$ respectively low and high enough, such that the $P_{d c_{l i m}}$ is hit before hitting $u_{d c_{\min }}$ or $u_{d c_{\max }}$, no voltage limits are active. Figs. $12-13$ shows the results for the voltage droop control after an outage of converter 2, assuming a 1 p.u. power change corresponding to a $10 \%$ voltage drop/increase. The results were respectively obtained using MatDyn and EUROSTAG. EUROSTAG uses an advanced symmetrically A-stable algorithm with variable step size that guarantees constant accuracy as well as high speed simulation [26]. In these simulations, the control scheme continues operating as a voltage droop scheme, since no voltage limits were hit at any of the converters. Similarly to the results in case of the voltage margin control in Fig. 9, converter 1 hits a current limit, which is accounted for by the other converter's droop

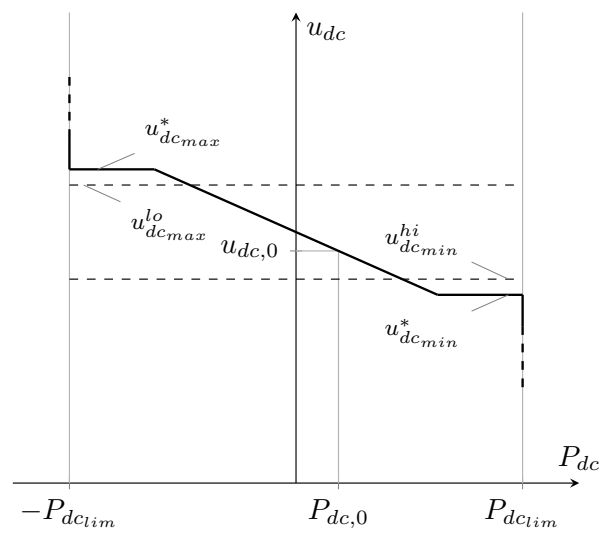

Fig. 14. Voltage droop control: P-V diagram.

action. The advantage of this voltage droop over the voltage margin control, is that the power after the converter outage is shared amongst the different droop controlled converters in the DC system, which makes the voltage droop control a suitable candidate for an operation in large DC grids. The joint control action of the different converters in the system also results in smaller voltage deviations.

\section{REDUCED ORDER MODELS}

This section discusses to what extent it is possible to further reduce the order of the model by disregarding some of the dynamics in the system. As discussed in [27], the overall 


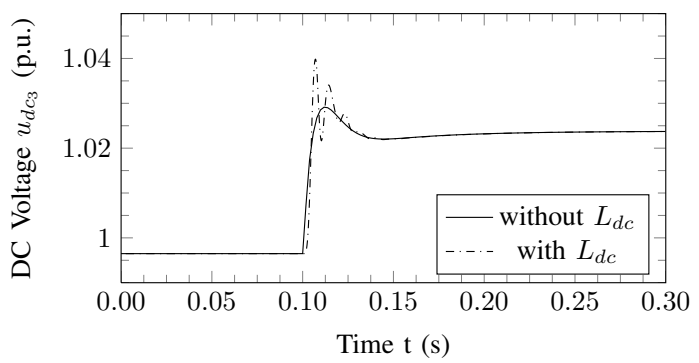

Fig. 15. Voltage droop control after outage of converter 2: Effect of including $L_{d c}$ on $u_{d c_{3}}$ (MatDyn simulation).

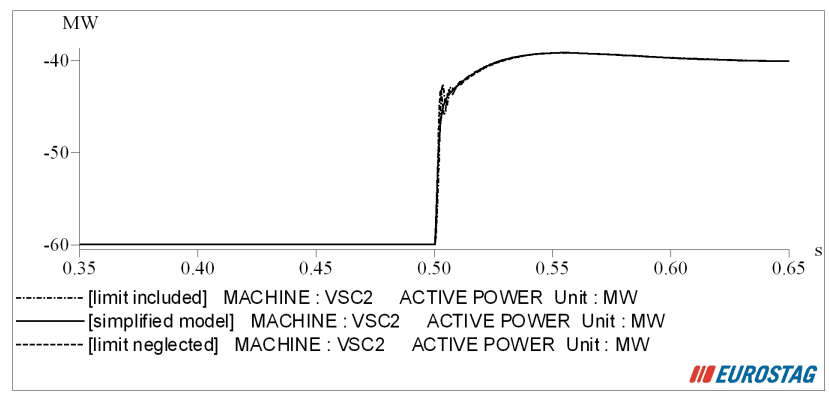

Fig. 16. Small active power step change in a two-terminal system.

order of the DC system can be reduced by eliminating one or more state variables. The models presented in this paper so far neglected the actual switching dynamics but included all controls and limits. In transient stability programs, the AC system is modelled by a set of steady-state equations, thereby neglecting all network electrical transients. The remainder of the paper will address modifications that can be made in the presented model and gives a rationale for these simplifications, as well as some considerations when more elaborated models should be used. A first part focuses on the DC grid dynamics, a second part addresses the converter control.

\section{A. DC grid model}

In Fig. 3, the DC lines are modelled using $\pi$-equivalent schemes. This lumped representation incorporates the most significant DC oscillations. When interactions of the DC circuit and the converter control or switching harmonics are of interest, more accurate frequency-dependent cable models, as used in [28], combined with detailed switching models are more appropriate.

When, on the contrary, the DC grid model is intended to be used in AC transient stability software, the question arises to what extent the DC dynamics have to be represented. This observation is justified by the fact that it is common practice to disregard all electromagnetic transients in the $\mathrm{AC}$ system as well. But contrary to the phenomena studied in the AC system in transient stability studies, the converter control interactions that are included in the DC model are determined by the voltage and current dynamics in the DC system.

In two-terminal systems, it is common practice to represent the DC network by one single DC bus with an equivalent capacitance. In this way, the overall DC grid voltage dynamics

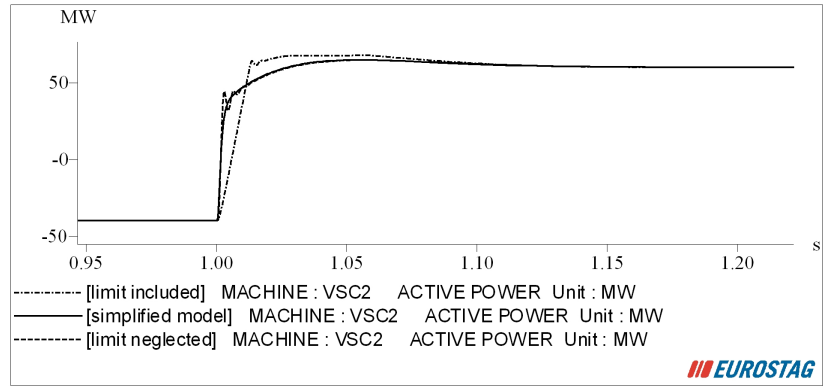

(a)

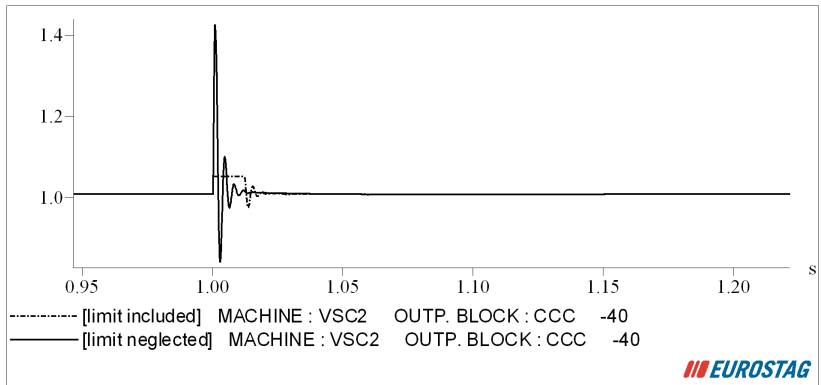

(b)

Fig. 17. Large active power step change in a two-terminal system: (a) Active power $P_{s}$ and (b) converter voltage $u_{c q}$.

used to tune the DC voltage controller are incorporated in the model. However, this model reduction does not reveal any information on the power flows in the DC system before and after a contingency, which can be an oversimplification when modelling DC grids. It also impedes to determine the actual DC voltage droop control settings: although the overall voltage dynamics are preserved, the DC voltage profile that leads to different voltage setpoints for all converters is not accounted for.

Alternatively, the model can be reduced by disregarding the DC line inductances. This eliminates the DC line currents as state variables in (6) while keeping the DC voltages as state variables. Contrary to what one would expect, eliminating the inductances can decrease the minimum step-size of the solver as the DC dynamics are then dictated by the time constants $R_{d c} C_{d c}$. In the results presented in the previous part, $L_{d c}$ has been respectively included for the MatDyn results (Figs. 9 and 12) and neglected for the EUROSTAG results (Fig 13). Fig. 15 show the effect of neglecting the DC inductances for the DC voltage at converter 3 when under voltage droop control. Fig. 15 shows the results for MatDyn. Including the inductances in EUROSTAG led to similar results.

\section{B. Converter control reduction}

As suggested in [27], the converter dynamics can be easily neglected by eliminating the first order system representing the converter switching from Fig. 2. The possibilities to reduce the order of the converter and controller model further is somewhat limited by the presence of the current and voltage limits. Although these converter limits can be disregarded when undertaking small signal stability studies, they are crucial with respect to the dynamic response and steady-state 


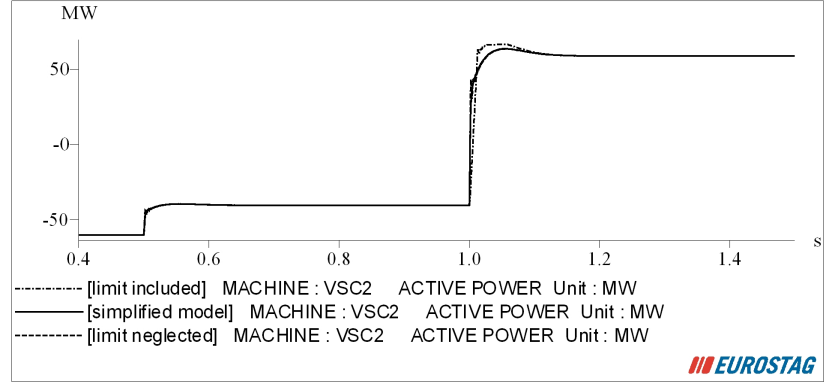

Fig. 18. Active power step changes: Full simulation results.

working conditions of the system. This can be illustrated by observing the dynamics incorporated by the voltage limits.

When approximating the current controller by an equivalent first-order system, these voltage limits are no longer taken into account. A way of dealing with this is limiting the reactive power reference, which can be written in function of the voltage limits. While this should give correct results for the steady-state values, the dynamics could be different: one would expect a slower response when the voltage limit is activated.

This is illustrated in a simulation example. A $20 \%$ active power step at $\mathrm{t}=0.5 \mathrm{~s}$, and a $100 \%$ active power step at $\mathrm{t}=1.0 \mathrm{~s}$ is simulated. The simulation has been performed for three different models: the model with voltage limit included, the model with voltage limit neglected, and the model with simplified current controller. In Fig. 16, a close up around $\mathrm{t}=0.5 \mathrm{~s}$ is shown. For the $20 \%$ step, the results of the three models correspond well. In Fig. 17, a close up around $\mathrm{t}=1.0 \mathrm{~s}$ is shown. For this larger step, it can be clearly seen that the dynamic behavior of the model with voltage limit is different. The rise time is slower and the maximum value of power in the first swing is higher (73 MW vs. $55 \mathrm{MW}$ ). This is not captured in the simplified model. The lower graph shows the voltage, which is limited at 1.05 p.u. for the model with limit. The full simulation is shown in Fig. 18. It is left to the appreciation of the reader if and in which situation neglecting the voltage limit is acceptable.

Similar to the voltage limits, the current limit also has its impact on the steady-state operation and the dynamic response. The effect on the steady-state operation can be observed from Figs. 9 and $12-13$, where converter 3 hits a current limit.

\section{CONCLUSION}

In this paper, a general electromechanical multi-terminal VSC HVDC model has been presented. The salient features of the model are that it has a generalized cascaded control scheme for MTDC systems that allows for voltage margin and voltage droop control and that current and voltage limits are represented in detail. A strategy to cope with a loss of the voltage controlling converter in a two-terminal system is generalized to voltage margin control for multi-terminal system and the model has been extended to include distributed DC voltage control. It is shown by simulations how the limits influence the dynamics and what the effects are of neglecting converter limits. The results indicate that reduced order models approximate the detailed model well. The model has been implemented in the open-source, MATLAB-based shell, MatDyn, as well as in a commercial power system simulation software, EUROSTAG, and has been tested on a four-terminal VSC HVDC system.

\section{REFERENCES}

[1] D. Van Hertem and M. Ghandhari, "Multi-terminal VSC HVDC for the European supergrid: Obstacles," Renewable and Sustainable Energy Reviews, vol. 14, no. 9, pp. 3156-3163, Dec. 2010.

[2] S. Cole, J. Beerten, and R. Belmans, "Generalized dynamic VSC MTDC model for power system stability studies," IEEE Trans. Power Syst., vol. 25 , no. 3, pp. 1655-1662, Aug. 2010.

[3] L. Zhang, L. Harnefors, and H.-P. Nee, "Power-synchronization control of grid-connected voltage-source converters," IEEE Trans. Power Syst., vol. 25, no. 2, pp. 809-820, May 2010.

[4] L. Zhang, H.-P. Nee, and L. Harnefors, "Analysis of stability limitations of a VSC-HVDC link using power-synchronization control," IEEE Trans. Power Syst., vol. 26, no. 3, pp. 1326-1337, 2011.

[5] T. M. Haileselassie, M. Molinas, and T. Undeland, "Multi-terminal VSC-HVDC system for integration of offshore wind farms and green electrification of platforms in the North Sea," in Proc. NORPIE 2008, Espoo, Finland, Jun. 19-21, 2008, 8 pages.

[6] N. R. Chaudhuri and B. Chaudhuri, "Adaptive droop control for effective power sharing in multi-terminal DC (MTDC) grids," IEEE Trans. Power Syst., vol. 28, no. 1, pp. 21-29, Feb. 2013.

[7] M. Aragüés-Peñalba, A. Egea-Àlvarez, O. Gomis-Bellmunt, and A. Sumper, "Optimum voltage control for loss minimization in HVDC multi-terminal transmission systems for large offshore wind farms," Electric Power Systems Research, vol. 89, pp. 54-63, 2012.

[8] E. Prieto-Araujo, F. D. Bianchi, A. Junyent-Ferre, and O. GomisBellmunt, "Methodology for droop control dynamic analysis of multiterminal VSC-HVDC grids for offshore wind farms," IEEE Trans. Power Del., vol. 26, no. 4, pp. 2476-2485, 2011.

[9] N. Chaudhuri, R. Majumder, B. Chaudhuri, and J. Pan, "Stability analysis of VSC MTDC grids connected to multimachine AC systems," IEEE Trans. Power Del., vol. 26, no. 4, pp. 2774-2784, Oct. 2011.

[10] C. Dierckxsens, K. Srivastava, M. Reza, S. Cole, J. Beerten, and R. Belmans, "A distributed DC voltage control method for VSC MTDC systems," Electric Power Systems Research, vol. 82, no. 1, pp. 54-58, Oct. 2012

[11] T. Haileselassie and K. Uhlen, "Primary frequency control of remote grids connected by multi-terminal HVDC," in Proc. IEEE PES GM 2010, Minneapolis, USA, Jul. 25-29, 2010, 6 pages.

[12] N. R. Chaudhuri, R. Majumder, and B. Chaudhuri, "System frequency support through multi-terminal DC (MTDC) grids," IEEE Trans. Power Syst., vol. 28, no. 1, pp. 347-356, Feb. 2013.

[13] J. Beerten, S. Cole, and R. Belmans, "Generalized steady-state VSC MTDC model for sequential AC/DC power flow algorithms," IEEE Trans. Power Syst., vol. 27, no. 2, pp. 821-829, May 2012.

[14] J. Beerten, D. Van Hertem, and R. Belmans, "VSC MTDC systems with a distributed DC voltage control - a power flow approach," in Proc. IEEE PowerTech '11, Trondheim, Norway, Jun. 19-23, 2011, 6 pages.

[15] T. M. Haileselassie and K. Uhlen, "Impact of DC line voltage drops on power flow of MTDC using droop control," IEEE Trans. Power Syst., vol. 27, no. 3, pp. 1441-1449, Aug. 2012.

[16] T. Nakajima and S. Irokawa, "A control system for HVDC transmission by voltage sourced converters," in IEEE PES Summer Meeting, 1999, vol. 2, 1999, pp. 1113-1119.

[17] T. Haileselassie, K. Uhlen, and T. Undeland, "Control of multiterminal HVDC transmission for offshore wind energy," in Proc. Nordic Wind Power Conference Presentation, Rønne, Denmark, Sep. 10-11, 2009, 7 pages.

[18] S. Cole and R. Belmans, "MatDyn, a new Matlab-based toolbox for power system dynamic simulation," IEEE Trans. Power Syst., vol. 26, no. 3, pp. 1129-1136, 2011.

[19] EUROSTAG website. [Online]. Available: http://www.eurostag.be

[20] A. Yazdani and R. Iravani, Voltage-Sourced Converters in Power Systems: Modeling, Control and Applications. Wiley, 2010.

[21] L. Zhang, "Modeling and control of VSC-HVDC links connected to weak AC systems," Ph.D. dissertation, Royal Institute of Technology, (KTH), Stockholm, Sweden, 2010.

[22] C. Feltes, H. Wrede, F. W. Koch, and I. Erlich, "Enhanced fault ridethrough method for wind farms connected to the grid through VSCbased HVDC transmission," IEEE Trans. Power Syst., vol. 24, no. 3 , pp. 1537-1546, Aug. 2009. 
[23] L. Zhang, L. Harnefors, and H.-P. Nee, "Interconnection of two very weak AC systems by VSC-HVDC links using power-synchronization control," IEEE Trans. Power Syst., vol. 26, no. 1, pp. 344-355, 2011.

[24] "It's time to connect - technical description of HVDC Light technology," ABB, Tech. Rep., Mar. 2008.

[25] MatACDC [Online]. Available: http://www.esat.kuleuven.be/electa/teaching/matacdc/

[26] J. Astic, M. Jerosolimski, and A. Bihain, "The mixed Adams-BDF variable step size algorithm to simulate transient and long term phenomena in power systems," IEEE Trans. Power Syst., vol. 9, no. 2, pp. 929-935, May 1994.

[27] S. Cole and R. Belmans, "A proposal for standard VSC HVDC dynamic models in power system stability studies," Electric Power Systems Research, vol. 81, no. 4, pp. 967-973, 2011.

[28] F. Mura, C. Meyer, and R. W. De Doncker, "Stability analysis of highpower DC grids," IEEE Trans. Ind. Appl., vol. 46, no. 2, pp. 584-592, Mar./Apr. 2010.

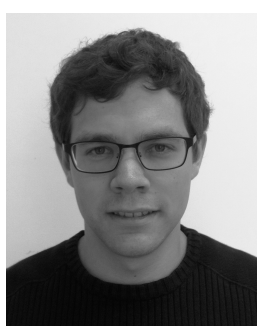

Jef Beerten (S'07-M'13) was born in Belgium in 1985. He received the M.Sc. degree in electrical engineering from the University of Leuven (KU Leuven), Leuven, Belgium, in 2008, and the Ph.D. degree in 2013, also from KU Leuven. $\mathrm{He}$ is a Postdoctoral Researcher with the ESAT-ELECTA division of KU Leuven. His research interests include power system control, the grid of the future and multiterminal VSC HVDC in particular. Mr. Beerten is an active member of both the IEEE and CIGRÉ.

Mr. Beerten's research has been funded by a Ph.D. fellowship from the Research Foundation - Flanders (FWO). Currently, he holds a postdoctoral fellowship from the FWO.

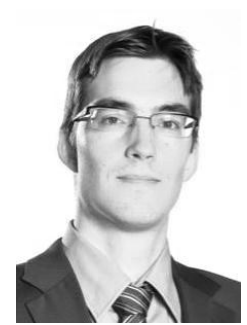

Stijn Cole (S'06-M'10) received the M.Sc. degree in electrical engineering in 2005 from the University of Leuven (KU Leuven), Belgium. After completing his doctoral dissertation on modelling and simulation of HVDC systems, he received the Ph.D. degree in 2010 from the same university. In 2010, he joined Tractebel Engineering where he is currently working as a power system consultant.

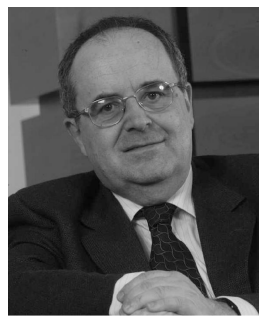

Ronnie Belmans (S'77-M'84-SM'89-F'05) received the M.Sc. degree in electrical engineering in 1979 and the Ph.D. degree in 1984, both from the University of Leuven (KU Leuven), Belgium, the Special Doctorate in 1989 and the Habilitierung in 1993, both from the RWTH, Aachen, Germany. Currently, he is a full professor with the KU Leuven, teaching electric power and energy systems. His research interests are within the framework of grids, smart grids, the deployment of all the new grid technologies and their integration with new resources and the demand in electricity. He is also Guest Professor at Imperial College of Science, Medicine and Technology, London, UK. Dr. Belmans is a Fellow of the IEEE and the IET. He is also the CEO of EnergyVille, the Executive Director of the Global Smart Grid Federation and Honorary Chairman of the board of Elia, the Belgian transmission grid operator. 